\title{
Individualizing dialysis dose under certain circumstances during the Severe Acute Respiratory Syndrome Coronavirus-2 (coronavirus disease 2019) pandemic: a perspective
}

\author{
Tarek Samy Abdelaziz \\ Department of Renal Medicine, Kasr Alainy University Hospitals, Cairo, Egypt
}

Severe Acute Respiratory Syndrome Coronavirus-2 (SARS-CoV-2) is the current major challenge to healthcare systems. The virus broke out in Wuhan, China, and quickly spread across the globe. The pandemic poses high risk to the whole world; experts are foreseeing more than one wave of the outbreak. The challenges to healthcare systems amid this pandemic are massive. Understaffing due to sick leave is a significant concern. Shortages of medical supplies have resulted due to demandproduction mismatches [1].

Hemodialysis may confer an additional risk due to the nature of renal replacement therapy that requires attending the dialysis clinic 3 times weekly. It is not clear whether coronavirus disease 2019 (COVID-19) viral infection rates are higher in dialysis patients [1].

Several societies have endorsed guidelines for prevention of the pandemic in hemodialysis patients. The Chinese Society of Nephrology and he Taiwan Society of Nephrology endorsed specific recommendations for patients receiving hemodialysis. The European Dialysis (EUDIAL) working group of the European Renal Associ-

Received April 17, 2020; Revised May 25, 2020;

Accepted May 26, 2020

Editor: Young-Ki Lee, Hallym University, Seoul, Republic of Korea Correspondence: Tarek Samy Abdelaziz

Department of Renal Medicine, Kasr Alainy University Hospitals, Kasr Alainy Cairo 11562, Egypt. E-mail: taroukah5070@kasralainy. edu.eg

Copyright () 2020 by The Korean Society of Nephrology

(a) This is an open-access article distributed under the terms of the Creative Commons Attribution Non-Commercial License (http://creativecommons. org/licenses/by-nc-nd/4.0/), which permits unrestricted non-commercial use, distribution, and reproduction in any medium, provided the original work is properly cited.
ation-European Dialysis has endorsed similar recommendations [2]. For healthcare workers, the recommendations highlight the importance of personal protection and proper infection control measures to avoid cross infection. For patients on dialysis, the core of recommendations is to avoid personal contact and travel except for attending the dialysis centers, accompanied by proper hand hygiene, reporting any new symptoms of cough, shortness of breath, or fever to the medical staff.

The concept of incremental dialysis may be suitable for some patients amid the widespread pandemic. This point of view has been expressed by experts around the world $[1,3]$. The recommendations entail gradual initiation of hemodialysis rather than full-dose dialysis, increasing the dose of dialysis incrementally with decline of residual kidney function [4]. Incremental hemodialysis involves twice weekly or even once weekly hemodialysis. Hemodialysis patients who become infected with SARSCoV-2 are not the target of this recommendation, as the exact dose and modality of dialysis associated with favorable outcome in this context is not known. A metaanalysis concluded that patients on incremental hemodialysis had no increased mortality compared to patients started on full dose dialysis [4]. There are many barriers to optimal utilization of incremental dialysis, including non-predictability of the decline of residual kidney function, non-adherence of patients in performing repeated measurement of residual kidney functions, and lack of randomized controlled trials [5]. Those limitations could be overcome by applying stringent criteria and launching clinical trials comparing incremental dialysis to thrice weekly.

In China, it was estimated in 2013 that about $26 \%$ of 
Table 1. Suggested criteria for individualization of dialysis dose during the SARS-CoV-2 pandemic

\begin{tabular}{l} 
Patients characteristics \\
1) Clinical parameters: euvolemic status and good blood pressure \\
control in the previous months \\
AND \\
2) Laboratory parameters: target hemoglobin, calcium, and \\
phosphorus in line with Kidney Disease Improving Global Outcome \\
guidelines for management of patients on hemodialysis \\
AND \\
3) Good dietary compliance \\
AND \\
4) Residual kidney function \\
AND \\
5) Deemed high risk of infection due to old age (> 70 years) or other \\
factors \\
Center characteristics \\
Centers experiencing higher than usual flow of patients or where \\
the service has been interrupted by staff absenteeism or under- \\
resourcing \\
\hline SARS-CoV-2, Severe Acute Respiratory Syndrome Coronavirus-2.
\end{tabular}

patients receive twice daily dialysis. Although $\mathrm{Kt} / \mathrm{v}$ was lower than that of China Dialysis Outcomes and Practice Patterns Study countries, the quality of life was similar between patients on twice weekly dialysis to those on a three times weekly schedule [6].

In Egypt, like many other counties, incremental dialysis is an evolving practice that still needs verification through randomized controlled trials [7]. Unsurprisingly, no specific guideline addresses the appropriate timing and dose of incremental dialysis or selection criteria other than residual kidney function.

Renal physicians should apply stringent selection criteria for patients who might be eligible for this approach. Table 1 shows the characteristics of such patients. Residual kidney function requires preservation of $>0.5 \mathrm{~L} /$ day urine output. Ten criteria have been proposed previously as prerequisites to start incremental dialysis [8]. Elderly patients who receive hemodialysis at clinics are particularly at increased risk. The mortality rate of COVID-19 is increased in the elderly. Such elderly patients with residual renal function may be candidates for incremental dialysis on case by case basis. The benefit for this vulnerable cohort of patients is to minimize exposure to infections. It is of utmost importance to ensure that restoration of thrice weekly dialysis will occur if any complications are anticipated.
Individualization of dialysis dose for selected patients might have a beneficial effect on healthcare facilities. The overworked dialysis staff will have fewer tasks and will be able to provide better quality service to dialysis patients.

The main advantage of this approach is minimized risk of cross infection for patients on hemodialysis, where clinical and laboratory parameters allow judicious reduction in the weekly dialysis dose.

There is an expected substantial decrease to the number of patients receiving kidney transplantation during this epidemic. This will lead to subsequent increase in patients initiating renal replacement therapy while awaiting delayed transplantation.

In conclusion, the COVID-19 pandemic poses risk to patients receiving 3 times weekly hemodialysis. Alternative strategies are required to address the resourcesdemand mismatch at dialysis facilities. Shortage of medical supplies and understaffing are important considerations. The judicious practice of individualization of dialysis dose at this challenging time may reduce the risk of exposure for risk patients with suspected or confirmed COVID-19. Moreover, this practice may provide capacity for dialysis facilities to accommodate new patients as a consequence of delayed kidney transplant surgeries. The cases for this protocol should be based on stringent criteria and sound clinical judgment.

\section{Conflicts of interest}

The author has no conflicts of interest to declare.

\section{References}

[1] Ikizler TA, Kliger AS. Minimizing the risk of COVID-19 among patients on dialysis. Nat Rev Nephrol 2020;16:311313.

[2] Basile C, Combe C, Pizzarelli F, et al. Recommendations for the prevention, mitigation and containment of the emerging SARS-CoV-2 (COVID-19) pandemic in haemodialysis centres. Nephrol Dial Transplant 2020;35:737-741.

[3] Meyer TW, Hostetter TH, Watnick S. Twice-weekly hemodialysis is an option for many patients in times of dialysis unit stress. J Am Soc Nephrol 2020;31:1141-1142.

[4] Garofalo C, Borrelli S, De Stefano T, et al. Incremental dialysis in ESRD: systematic review and meta-analysis. $J$ Nephrol 2019;32:823-836. 
[5] Murea M, Moossavi S, Garneata L, Kalantar-Zadeh K. Narrative review of incremental hemodialysis. Kidney Int Rep 2019;5:135-148.

[6] Yan Y, Wang M, Zee J, et al. Twice-weekly hemodialysis and clinical outcomes in the China dialysis outcomes and practice patterns study. Kidney Int Rep 2018;3:889-896.

[7] Elrggal ME, Zyada R. Gradual initiation of dialysis as a means to reduce cost while providing quality health care. Nat Rev Nephrol 2017;13:720.

[8] Kalantar-Zadeh K, Unruh M, Zager PG, et al. Twice-weekly and incremental hemodialysis treatment for initiation of kidney replacement therapy. Am J Kidney Dis 2014;64:181186. 